\title{
Estrategia educativa para el desarrollo de la motivación profesional en los estudiantes de sociología
}

\author{
Educative Strategy for the Development of \\ Professional Motivation in Students of Sociology
}

\author{
Yudenia Nieves Basulto ${ }^{1}$ \\ Silvia Colunga Santos ${ }^{2}$
}

\section{Resumen}

La estrategia educativa que se presenta como resultado científico está orientada a fortalecer la motivación profesional del estudiantado de la carrera de sociología en condiciones de semipresencialidad en el Centro Universitario Municipal (CUM) de Vertientes, provincia de Camagüey. La misma incluye tareas a desarrollar dentro y fuera del aula, que permiten al estudiante conocer la carrera, sus modos de actuación, la vinculación con la especialidad desde su propia área de acción, la utilidad social de la profesión y a identificar los beneficios materiales y espirituales que se derivan del ejercicio de la misma. El estudiante, desde la mentoría, relacionará su proyecto de vida personal y profesional con la carrera que cursa y el futuro ejercicio de esta. Como resultado de la introducción de la estrategia en la práctica educativa universitaria, los alumnos asumirán, paulatinamente, un papel activo y serán orientados por los mentores y profesor guía en relación con su desarrollo profesional.

\section{Palabras clave}

Motivación profesional, mentoría, estrategia educativa.

\section{Abstract}

As a scientific result, this educative strategy was designed to encourage motivation of the part-time students of the Sociology career in the Municipal University Center of Vertientes. The strategy's main goals are promoting internal and extra activities that allow students to get interested in the career, its characteristics and the connections with their own specific area, as well as its social importance and the material and spiritual benefits related to this specialty. The student with the help of his mentor will be able to relate his professional and personal project with his own career and his future performance as a professional. Due to the application of this strategy in the university educative practice, the students will play gradually an active role in the way they will perform as professionals in the future. They will be also advised by their own mentors and guide professors taking into consideration this last aspect.

\section{Keywords}

Professional motivation, mentorship, educative strategy.

Artículo recibido el 2 de julio de 2014 y aprobado el 20 de agosto de 2014

1 Centro Universitario Municipal de Vertientes, Camagüey, Cuba. Correo electrónico: yudenia.nieves@reduc.edu.cu

2 Centro de Estudios de Ciencias de la Educación, Universidad de Camagüey, Cuba. Correo electrónico: silvia.colunga@reduc.edu.cu 


\section{Introducción}

En el mundo actual, la educación superior tiene como misión esencial formar profesionales con una alta calificación, que actúen de forma responsable y competente, lo cual significa trascender el modelo del profesional con conocimientos y habilidades profesionales para sustituirlo por una concepción en la que se conciba como un individuo capaz de orientar su actuación con independencia y creatividad a partir de una sólida motivación hacia la profesión. Es por ello que la enseñanza universitaria debe promover no solo el aprendizaje de conocimientos y habilidades profesionales, sino también la formación de motivos de actuación que conduzcan al desarrollo profesional y al crecimiento personal, siendo la orientación profesional el proceso educativo que puede conducir al logro de tan importantes objetivos.

La carrera de sociología, particularmente, se estudia por curso diurno en las universidades de La Habana y Oriente. Cuando acontece el surgimiento de las sedes universitarias municipales, se universalizan los estudios de la misma, sin existir una experiencia previa de orientación profesional. La carrera se ha caracterizado, además, por contar con pocos profesionales en cada provincia y se ha divulgado poco sobre la labor del sociólogo, existiendo centros de investigaciones sociológicas únicamente a nivel nacional. Es una especialidad que muchas veces las personas desconocen. Estas particularidades hacen que el proceso de orientación profesional previo a la selección de la carrera, se entorpezca.

A partir de investigaciones realizadas por las autoras en la carrera de sociología en el Centro Universitario Municipal de Vertientes, se ha podido constatar la existencia de insuficiencias en el proceso de orientación profesional en los estudiantes. Estas se expresan en el escaso conocimiento que poseen con respecto a elementos indispensables en su formación: objeto de estudio, perspectivas, aplicabilidad social de su especialidad, entre otros. Además, poseen un pobre vínculo afectivo con la carrera, los profesores y tutores no conocen a cabalidad detalles de la especialidad por carecer de formación sociológica, no se promueve de manera suficiente la prác- tica investigativa en la carrera relacionada con el entorno del estudiante, lo cual provoca que el mismo no perciba los nexos entre la teoría y la práctica. Los estudiantes se apropian del conocimiento de manera momentánea, para una evaluación, pero no para su aplicabilidad en la práctica profesional.

Dentro de ese proceso de orientación profesional del sociólogo, la motivación hacia la profesión tiene una connotada importancia, por cuanto se convierte en impulsora de un modo de actuación adecuado, en correspondencia con las exigencias de la profesión. El presente trabajo es contentivo de una estrategia educativa para fomentar en los estudiantes la motivación por la profesión a partir de la tutoría como forma de organización de la enseñanza que puede tributar al logro de niveles de motivación por la profesión adecuados. Consta de varias etapas donde se incluyen actividades para mentorizar a los estudiantes de los últimos años que, guiados por los profesores tutores, contribuirán a reafirmar los motivos por la profesión de los estudiantes de los primeros años, revirtiéndose en resultados halagüeños para todos. Incluye, además, talleres que, de manera simultánea, se realizaron como soporte al objetivo fundamental de la estrategia educativa.

\section{Desarrollo}

En la educación superior cubana la orientación educativa adquiere un gran valor, tiende al desarrollo integral de la personalidad, con un carácter armónico, integral y consciente. Abordar esta cuestión desde una óptica educativa constituye un reto en el nuevo contexto mundial que hoy se vive. El papel orientador que pueden ejercer los profesores tutores en el contexto de la nueva universidad cubana cobra una gran importancia, recabándose del mismo para dotar al individuo-discente de la preparación requerida para salir adelante sin grandes frustraciones, de los conflictos que deberá enfrentar. Admitir esta posición implica reconocer la orientación como un proceso continuo, dinámico, planificado, potenciador de capacidades humanas; capaces de lograr en el individuo la compatibilidad de sus aspiraciones personales y las necesidades sociales. 
En este sentido, la escuela es planteada como la institución esencial para encauzar la orientación educativa de acuerdo con propósitos y fines determinados. Supone los elementos siguientes:

- La educación, toda ella, puede verse bajo el prisma de la orientación: la educación es orientación en cuanto que preparación para la vida, la cual se desarrolla en un itinerario personal que determinan circunstancias externas, pero que, en alguna medida, las opciones propias de cada persona contribuyen también a determinar.

- La orientación puede verse, por otra parte y además, como asesoramiento sobre caminos diferentes y sobre las correspondientes opciones que pueden emprender las personas.

- La orientación significa también educar en la capacidad para tomar decisiones propias, sobre todo aquellas que tienen una mayor trascendencia personal o que comprometen el futuro del tutelado.

- La orientación psicopedagógica consiste, bajo un cuarto aspecto, en la preparación de los alumnos para dirigir sus propios procesos de aprendizaje, en favorecer la adquisición de estrategias para aprender a aprender. Una parte de las mismas se recoge bajo el nombre de metacognición. Otra corresponde a las estrategias de control y manejo de procedimientos de aprendizaje: ahí se incluyen desde las técnicas de estudio, hasta las capacidades de aprender a aprender, de búsqueda y organización de la información pertinente, de automonitorización, autocorrección, autoevaluación y, en general, de aprender por uno mismo (Colunga, 2009b, p. 15).

Desde la óptica de diversos investigadores, el profesor juega un papel muy importante en la orientación profesional. Al ser un observador por excelencia, puede determinar cuáles estudiantes precisan más o menos orientación sobre la carrera o profesión. Estos argumentos constituyen una dirección importante del trabajo porque la orientación experta potencia la influencia educativa de los estudiantes. La estructuración de un programa de orientación con una concepción integradora garantiza la universalidad, la coherencia y la continuidad del proceso de orientación.
Este proceso de orientación profesional -que tiene que ver con el desarrollo de la esfera motivacional-afectiva y cognitivo-instrumental de la personalidad del estudiante universitario, es decir, de conocimientos, habilidades, motivos e intereses profesionales - también se relaciona con la esfera autovalorativa del sujeto y de cualidades de la personalidad tales como la independencia, la perseverancia, la flexibilidad; que le posibiliten una actuación profesional autodeterminada.

En Cuba se han definido principios que rigen la concepción e instrumentación de la orientación profesional en los centros universitarios, siendo imprescindibles:

- El enfoque profesional del proceso de enseñanza-aprendizaje. Esto implica que todo el proceso pedagógico debe ser organizado en función de la profesión, todas las actividades, tanto académicas como investigativas, deben responder a la profesión.

- El reconocimiento del estudiante como sujeto activo de su formación profesional. En virtud de este principio se considera que el estudiante es un ente protagónico en el proceso de su formación profesional, lo cual implica diseñar situaciones de aprendizaje que fomenten la iniciativa, la independencia y la responsabilidad del alumno en la realización de tareas de contenido profesional.

- El año como célula básica para la orientación profesional. En este caso se concibe al año académico como el espacio donde se integran los componentes académicos, investigativos y laborales en el proceso de aproximación del alumno al objeto de la profesión (González, 2002).

Cuando se analizan los contextos universitarios actuales en Cuba, se comprende que el proceso de orientación profesional en los Centros Universitarios Municipales no acontece de la misma manera que en las universidades centrales. Esto se debe, entre otras razones, a la inclusión en las aulas universitarias de un mayor número de alumnos de diferentes edades y sectores sociales, en virtud de lo cual cada vez más jóvenes pero también adultos mayores optan por el estudio de las diferentes profesiones que se ofrecen en el país. 
Aparejado a este renovador concepto de calidad, se habla de términos como pleno acceso, permanencia y egreso, los cuales a su vez se relacionan, entre otros aspectos, con el perfeccionamiento de la labor educativa y político ideológica; lo que presupone la consolidación del papel del profesor, la identificación precisa de las diferencias individuales de los estudiantes y la prioridad de la atención individualizada a esas diferencias.

Por otra parte, la universalización de la enseñanza ha impuesto nuevos retos al contexto universitario cubano relacionados, en lo fundamental, con la redefinición de saberes; la formación centrada en el autoaprendizaje, la autoeducación, la creatividad, flexibilidad y capacidad de adaptación a los cambios; el desarrollo de competencias que permitan resolver problemas profesionales y la formación integral centrada en el desarrollo de valores.

La flexibilidad de las condiciones en que se forma al estudiante, propiciando así una dinámica menos presencial en el proceso, ha hecho emerger con fuerza la figura del profesor-tutor, en cuyo trabajo se integra el sistema de relaciones básicas que se establecen a partir de la asesoría académica e investigativa de los profesores de las diferentes asignaturas, la extensión universitaria, el colectivo laboral al que pertenece el educando y la orientación hacia la profesión que estudia.

Esto exige entender la figura del tutor como el eje integrador que compatibiliza las responsabilidades laborales de los estudiantes con su formación profesional, personal, laboral y social. Se constituye entonces en el centro de influencias educativas con potencialidades de desarrollar la motivación profesional, siendo la figura del tutor y su quehacer pedagógico un requisito importante para lograr el profesional integral y comprometido con su profesión, indispensable en los tiempos actuales.

La estrategia educativa que se presenta parte del reconocimiento del profesor tutor como factor clave en el desarrollo de la motivación profesional del sociólogo en formación en condiciones de semipresencialidad. Atendiendo a la diversidad del alumnado que caracteriza este modelo de continui- dad de estudios, se nutre además de las ventajas que significa la labor del tutor atendiendo a que forma parte de un proceso continuo, sistemático y que se desarrolla de forma activa y dinámica, lo que explica su flexibilidad y la diversidad de contextos en los que tiene lugar.

Desde el punto de vista sociológico, la estrategia se basa en el encargo social que tiene la escuela como eje de influencias educativas e instructivas, así como en la importancia de la labor de los educadores. Se atiene al hecho de que en la sociedad cubana actual, se demanda cada vez más de un profesional altamente motivado e identificado con su quehacer profesional. Por ende, la universidad como eje de influencias socializadoras que responden a ese encargo social y como responsable de la formación profesional, necesita que de sus aulas egresen profesionales altamente competentes para las tareas que les aguardan, tanto en el plano de los conocimientos como en lo relacional afectivo.

Dentro de los presupuestos psicológicos tenidos en cuenta para la formulación de la estrategia educativa que se presenta, las autoras se identifican con el enfoque histórico-cultural de L. S. Vigostky y sus seguidores, sobre todo en lo concerniente al carácter mediatizado de los procesos psíquicos; la unidad de lo cognitivo y afectivo en la personalidad. Desde esta óptica que guía la escuela cubana actual, el estudiante es visto como objeto y sujeto de su aprendizaje, que asume una participación activa y responsable en su propio proceso de formación, mientras que al profesor se le confiere el rol de guía o conductor del proceso docente-educativo. También fue tenido en cuenta el concepto vigostkiano de zona de desarrollo próximo, donde se le concede un valor inestimable a todo lo que puede aprender el estudiante potencializado por el conjunto de influencias educativas del contexto educativo.

Dentro de la fundamentación teórica se destacan las concepciones de intenciones e intereses profesionales de Fernando González Rey y Viviana González Maura. González Rey refiere que las intenciones profesionales son 
El nivel superior de desarrollo de la motivación hacia la profesión cuando ésta se convierte en una tendencia orientadora de la personalidad y el sujeto es capaz de estructurar de forma consciente su motivo profesional, en su vida presente y futura, basado en una valoración y fundamentación conscientes de sus propósitos en esta esfera. (F. González, 1982, p. 22)

Allí el autor agrupa los siguientes aspectos:

- Conocimiento del contenido de la profesión, de sus perspectivas, posibilidades de investigación de su objeto, aplicabilidad social, etc.

- Vínculo afectivo con el contenido de la profesión que se manifiesta en intereses concretos y una actitud emocional hacia la misma.

- Elaboración personal del contenido: aquí se analiza si el contenido expresado por el sujeto es a través de juicios y valoraciones, si está comprometido con la vida afectiva del sujeto o cuando es capaz de defender aquellos contenidos que forman parte de su elaboración personal (F. González, 1982, p. 24).

No obstante, el concepto de intereses profesionales ha sido abordado de manera más esclarecedora por Viviana González Maura en los estudios referidos a la motivación profesional. La autora establece tres niveles diferentes de integración de la motivación profesional:

Los niveles superior y medio de integración de la motivación profesional se caracterizan por la existencia de una formación motivacional específica que denominamos interés profesional, en cuya base motivacional predominan motivos intrínsecos al contenido de la profesión (motivos cognoscitivos) $y$ que en el orden funcional puede expresarse en diferentes niveles de complejidad en la medida que alcanza o no la condición de tendencia orientadora de la personalidad. (González, 2009, p. 2)

La misma autora esclarece que en el nivel superior, el interés profesional se manifiesta como intenciones profesionales, y en el nivel medio como intereses cognoscitivos hacia el estudio de la profesión. El nivel inferior de integración de la motivación profesional se caracteriza por la existencia de formaciones motivacionales diversas que participan en la regulación de la actividad profesional en cuya base motivacional predominan motivos extrínsecos al contenido de la profesión (personales o sociales) y que en el orden funcional no constituyen tendencias orientadoras de la personalidad. Declara, a su vez, que las diferencias en la expresión funcional de los intereses profesionales se manifiestan no solo en la posibilidad de su existencia o no como tendencia orientadora de la personalidad; sino además en la calidad de la integración funcional de los motivos que conforman su base motivacional. Es así que, cuando el interés profesional se expresa a través de intenciones profesionales, se manifiesta una plena integración de los diferentes motivos que conforman su base motivacional, toda vez que se logra su expresión reguladora de la actividad a través del contenido de la profesión.

Ambos autores plantean que la motivación profesional puede convertirse en una tendencia orientadora de la personalidad. Cuendo esto sucede, los motivos se expresan en una intención profesional o en intereses profesionales. Ambas categorías sirvieron de base para el diagnóstico realizado, así como de criterios para determinar el nivel de desarrollo de la motivación profesional.

Dentro de los referentes pedagógicos se tuvo en cuenta la unidad de los procesos cognitivos y afectivos como principio de la formación. Desde la concepción del enfoque histórico-cultural (de naturaleza dialéctica) los procesos motivacionales solo pueden estudiarse considerando que en su esencia está la unidad de lo cognitivo y lo afectivo y, sobre todo, que los niveles superiores de regulación de la motivación en cualquier esfera se alcanzan con el fortalecimiento de esta unidad.

Se asume el principio de la tutoría como forma organizativa del proceso docente educativo en la educación superior. Se parte de criterios vinculados a la labor del tutor dentro del contexto universitario: la actividad y la comunicación a modo de principios metodológicos en la comprensión y exploración de la personalidad, el carácter sociohistórico de la 
personalidad como expresión dialéctica del sistema individuo-personalidad, además de la orientación educativa a manera de una relación de ayuda en el proceso de tutoría dirigido a la formación profesional de los estudiantes universitarios.

Resulta imprescindible asumir el principio relativo a la atención a las diferencias individuales. La necesidad de brindar por los tutores un tratamiento diferenciado, estrictamente personalizado, a los sociólogos en formación, específicamente en materia de diagnóstico y seguimiento de la motivación profesional. Se hace necesario fomentar los motivos profesionales desde el contexto de su formación, atendiendo a las individualidades constitutivas del grupo.

El núcleo de la estrategia es la mentoría como forma de organización de la tutoría y no resulta novedoso en el escenario universitario de otros países: Inglaterra, Estados Unidos, España, Canadá y Australia, entre otros. Está ampliamente divulgada en los ámbitos educativos anglosajones (bajo el nombre de peer tutoring), y está considerada por la Unesco y por los expertos en educación como un método didáctico de alta efectividad, extraordinariamente útil para atender la diversidad y para conseguir una enseñanza de calidad. Es denominada mentoría o tutoría entre iguales. Ha constituido una alternativa para fortalecer los motivos profesionales desde la tutoría. Consiste en una modalidad donde los estudiantes del mismo curso o cursos superiores son los que guían, asesoran o ayudan en su adaptación, integración y aprendizaje a otros estudiantes, en diferentes momentos de su formación universitaria.

La tutoría entre iguales es un método de aprendizaje cooperativo, a través del cual un alumno (el alumno tutor) aprende enseñando a su compañero (el alumno tutorado), y este, a su vez, aprende gracias a la ayuda personalizada y permanente que recibe del alumno tutor. Así pues, es una opción que aprovecha pedagógicamente las diferencias entre los alumnos y permite ver la diversidad como un recurso y no como un problema.
La premisa que guía la utilización de esta modalidad de tutoría es, por un lado, que se trata de un asesoramiento entre compañeros, reduciendo la distancia, reforzando la empatía. Y por otro lado, el conocimiento que tiene el alumno veterano de la institución, de la carrera y del profesorado. De esta manera pueden ofrecer sus experiencias para facilitar la adaptación y resolución de problemas de diferente naturaleza que tienen otros compañeros.

Aunque sirve de base a la presente estrategia educativa, esta no está concebida en iguales términos que en otros países del mundo como España, Canadá u otros. Es imprescindible remitirse a los nuevos escenarios universitarios de Cuba; mucho más, a las condiciones de un Centro Universitario Municipal. En estas nuevas condiciones, la acción tutorial alcanza una influencia inestimable. Puede constituir la mentoría, adecuada al trabajo del CUM, una herramienta innovadora eficaz. La presente estrategia educativa tiene como objetivo general contribuir al desarrollo de la motivación profesional en los estudiantes de la carrera de Sociología.

Los objetivos específicos de la estrategia son: valorar la importancia de la carrera para el proyecto de vida personal y profesional de los sociólogos en formación; profundizar en el conocimiento acerca de la carrera que cursa el estudiante, mediante diferentes actividades y tareas y desarrollar el vínculo afectivo con la profesión.

La población a la que está dirigida la estrategia es la totalidad de los estudiantes de la carrera de sociología en el Centro Universitario Municipal de Vertientes. La variable principal a considerar es el desarrollo de la motivación profesional. Para el estudio de la motivación profesional se adoptaron como indicadores los propuestos por Fernando González Rey: el conocimiento del contenido de la profesión (la sociología en este caso), el vínculo afectivo con el contenido de la profesión y la elaboración personal del contenido.

\section{Estructura de la estrategia educativa}


La red de mentores está basada en una estructura de tipo piramidal en cuyo vértice se encuentra el coordinador general del proyecto, que es el único sociólogo existente en la carrera. La base está compuesta por los estudiantes mentorizados de la carrera de sociología. Los elementos o figuras que integran la estructura son de dos tipos: los que brindan información, experiencias, trabajo en beneficio de otros (alumnos de $5 .^{\circ}$ y $6 .^{\circ}$ años en compañía de profesores de la carrera y tutores) y los perceptores o tributarios de la ayuda, destinatarios últimos de la acción tutoral (en este caso, los estudiantes de $3 .^{\circ}$ y $4 .^{\circ}$ años).

En el primer grupo están los que facilitan o ejercen la relación de ayuda, en términos de coordinación, formación y asesoramiento. También forman parte de este grupo los profesores tutores que ejercerán una función de asesoramiento y formación de los alumnos mentorizados. Su misión consiste en desarrollar sesiones de formación con los mentores $y$, tras ese período, tutorizarlos durante todo el proceso de mentorización. Se harán cargo de los estudiantes de $3 .^{\circ}$ y $4 .^{\circ}$ año de la carrera, donde se encuentra la mayor cantidad de estudiantes. De esta manera se atenderán de forma más personalizada y eficaz, las demandas educativas y cognoscitivas de los alumnos en relación con la especialidad. A pesar de esta asignación inicial de los mentores o profesores tutores, quedará siempre abierta la posibilidad de comunicación de los mentores con los profesores tutores y el coordinador del proyecto.

En el segundo grupo se encuentran los beneficiarios o receptores de la relación de ayuda, es decir, los alumnos mentorizados. Al estudiante de estos años (3..$^{\circ}$ y.$^{\circ}$ años), se le exigen habilidades que se suponen haya ido adquiriendo desde los dos años anteriores. Se puede influir en el desarrollo de estas necesarias habilidades del sociólogo en formación así como en otros aspectos referidos a la aplicabilidad de la sociología en sus áreas de acción, consultas sobre aspectos de las asignaturas de esos cursos, experiencias vivenciales sobre la especialidad, entre otras disímiles situaciones espontáneas, las cuales los mentores pueden contribuir a resolver.
Es importante destacar que este proceso no debe ser visto como unidireccional, es decir, desde el mentor hacia el mentorizado; sino que a la par que ayuda, que educa y enseña al mentorizado, el mentor se nutre de conocimientos, se educa y aprende de esta relación de ayuda. El modelo de intervención que se utiliza se caracteriza porque la relación de ayuda entre el coordinador del proyecto y el beneficiario de la misma tiene a un mediador que es el mentor, estableciendo relaciones en ocasiones más directas y menos directas, en dependencia de las propias necesidades de los implicados en la estrategia educativa.

El profesional de ayuda es el profesor tutor, liderado por el coordinador del proyecto, que es quien asesora y forma a los mentores. El mediador es el mentor, objeto de asesoramiento y formación por parte del profesor tutor y coordinador. Este será quien después asesore e informe a los mentorizados. Por último, el beneficiario de la relación de ayuda es el alumno mentorizado, que recibe directamente la ayuda del mentor.

\section{Fases de la estrategia educativa}

Primera etapa: Aseguramiento de las condiciones para ejecutar la estrategia educativa. Es indispensable motivar a los estudiantes y profesores que participarán en la misma. Se necesita conseguir un ambiente estimulador y un clima afectivo que favorezca la reflexión y el diálogo mutuo.

Se sugiere para ello la implementación de un taller dividido en dos sesiones de aproximadamente dos horas de duración, dirigido en un primer momento a los profesores que intervendrán y posteriormente, otro taller destinado a los estudiantes. Se hará de manera diferenciada atendiendo a las especificidades de a quiénes va dirigido dicho taller.

En el taller dirigido a los profesores, la primera sesión perseguirá como objetivo fundamental que los docentes reflexionen acerca de la importancia de la motivación profesional para el desempeño académico del estudiantado. Dentro de los contenidos a tratar se encuentran aspectos referidos a la orientación profesional como proceso educativo necesario en el contexto universitario, y aspectos 
teóricos sobre la motivación profesional, actualidad de las investigaciones en materia de motivación profesional en Cuba.

Dentro de la bibliografía a utilizar se encuentran materiales que han servido de base al presente trabajo de investigación (González Rey, González Maura, Del Pino, Llerena Companioni, etc.). Es importante destacar la utilización de bibliografía, ya que muestra el carácter científico que tiene el proceso de motivación profesional en el escenario universitario, así como la actualidad que en el contexto educativo y social tiene.

La segunda sesión tendrá como objetivo fundamental unificar criterios sobre la aplicación y seguimiento de la estrategia educativa. En los contenidos a tratar se incluyen los fundamentos teóricos de la mentoría, surgimiento e implementación de la mentoría en otros países, contextualización de la mentoría en el CUM y, específicamente, en la carrera de sociología, fases de la implementación oportuna y gradual de la mentoría, compromiso de los docentes a la colaboración en la implementación de la mentoría.

El taller que está dirigido a los estudiantes también constará de dos sesiones. La primera tendrá como objetivo fundamental que los estudiantes analicen la importancia que tiene para su formación profesional la motivación por la carrera. Los contenidos a tratar serán el análisis de la motivación y de la motivación profesional, actualidad e importancia de los estudios relacionados con la motivación profesional e importancia de alcanzar niveles altos de motivación por la profesión en los futuros sociólogos.

La segunda sesión dirigida a los estudiantes tendrá como objetivo fundamental fundamentar la mentoría y su implementación en la carrera de sociología. Los contenidos a tratar serán surgimiento e implementación de la mentoría en otros países, contextualización de la mentoría en el CUM y, específicamente, en la carrera de sociología, así como fases de la implementación oportuna y gradual de la mentoría.

Es necesario en esta etapa recabar del papel activo que desempeñarán los estudiantes, ya que sus propios compañeros de últimos años serán los mentores, guiados por profesores tutores y el profesor guía. Se producirá un intercambio que permita conocer las expectativas que tienen de la mentoría y cómo tributará al logro de los objetivos propuestos su disposición para colaborar.

Segunda etapa: Diagnóstico inicial de los niveles de motivación profesional de los estudiantes de sociología en el CUM. En esta etapa se aplican los instrumentos de medida a todos los estudiantes de la carrera (44 alumnos en total), que serán la técnica de la composición, escalas valorativas, un completamiento de frases y un cuestionario, para utilizarlos no solo para corroborar la cuantía del problema objeto de investigación, sino también para comparar los resultados con los obtenidos en otro momento: en un momento intermedio o cuando culmine la aplicación de la estrategia.

Tercera etapa: Organización para la implementación de la estrategia educativa. Se realizará una reunión metodológica donde se estudiará con detenimiento todo el proyecto, se asignarán funciones y tareas a los miembros de los equipos y se establecerá la temporalización. Participarán los estudiantes y profesores. Se asignarán las funciones de profesores participantes, alumnos mentores y alumnos mentorizados.

Se debe infundir a los profesores y estudiantes de un espíritu cooperativo, colaborador, donde no primen las relaciones de superioridad, sino que todos sientan los beneficios de su implementación. Es necesario que perciban relaciones de armonía, colaboración, compañerismo, intercambio. En ese necesario encuentro, se organizarán las funciones de cada participante y se esclarecerán los detalles y dudas de todos.

Cuarta etapa: Implementación de la estrategia educativa. En esta fase se ejecutarán las actividades previstas. Se definirán relaciones verticales que fluirán de la siguiente manera: cada tutor trabaja con sus alumnos mentores constituyendo así los equipos tutor-mentor. Se reunirán una vez cada 15 días, aunque se sugiere que sea previo al día de los encuentros de los estudiantes. En este encuentro se atenderán las dudas y preguntas. Cada mentor tiene sus alumnos mentorizados seleccionados previa- 
mente en los grupos de $3 .^{\circ}$ y $4 .^{\circ}$ años de la carrera. Con los alumnos mentorizados los mentores se reunirán una vez a la semana, en un local fijo. No se descartan las relaciones horizontales para que la información fluya entre iguales, contribuyendo así a fortalecer el compañerismo, la camaradería, etc.

En esta etapa también se desarrolla el proceso de mentorización. Una vez identificados los estudiantes a mentorizar por los mentores, estos se dedicarán a la detección de necesidades profesionales de los mismos. Se garantiza así que todos los alumnos tutelados reciban la misma información sobre los temas que se consideran más importantes, abordándolos en el momento más adecuado.

A partir de las necesidades detectadas en investigaciones realizadas por las autoras, no se pueden obviar los siguientes temas: objeto de estudio de la sociología, surgimiento de la sociología como ciencia, áreas del conocimiento sociológico (educación, cultura, familia, política, trabajo, etc.), vinculación de los escenarios en que se desenvuelven los estudiantes con la sociología, el sociólogo como agente transformador de la realidad social, metodología de la investigación sociológica, presupuestos básicos, etc.

La estrategia no es cerrada. Al margen de los temas propuestos, es previsible que surjan nuevos temas y preguntas a tratar en cada reunión y equipo. Se pretende que la dinámica se ajuste lo más posible a las características de los grupos mentorizados.

Se sugiere un proceso en el que el mentor plantee una experiencia relacionada con la problemática a abordar que después será analizada a profundidad. El papel del mentor será facilitar, entre otras cuestiones, situaciones sociales que propicien el debate sobre el modo de actuación del sociólogo, el autorreconocimiento de la especialidad, la utilidad de los conocimientos aprendidos en el aula, etc. No es objetivo que los mentores se conviertan en conferencistas sobre sociología, hay que tener en cuenta que también son estudiantes en formación.

Se implementarán en las bibliotecas virtuales habilitadas en los laboratorios de computación de la institución educativa, materiales en soporte digital que puedan ser estudiados por los mentores, así como los mentorizados. Se realizarán las coordinaciones necesarias para que, atendiendo a los temas de interés que surjan, se pueda obtener información a través del correo electrónico con profesionales e investigadores de la temática social en otras instituciones del país, accediendo así a información actualizada. Para contribuir a la preparación de mentores y mentorizados, se sugieren talleres interactivos entre todos los miembros del proyecto, una vez al mes durante tres meses.

1. Taller "Conociendo mi carrera". Objetivo fundamental: identificar los diferentes aspectos que caracterizan la carrera que cursa, así como las razones por la que escogió la carrera que cursa. Entre las actividades a efectuarse encuentran: abordar investigaciones desarrolladas por sociólogos, relatar experiencias propias y ajenas sobrevivencias referidas a la carrera, propiciar el intercambio acerca de las razones que propiciaron la selección de la carrera. (Tiempo aproximado: 2 horas).

2. Taller "Me identifico con la carrera". Objetivo fundamental: identificar los aspectos más interesantes de la carrera de sociología, así como valorar las características propias de un profesional exitoso, motivado, competente. Entre las actividades se encuentran: entrevista a un profesional de la sociología de renombre en el ámbito de la localidad o invitado de otro lugar, exposición donde aborden qué debe caracterizar a un profesional motivado, visita a centros donde laboren profesionales de la especialidad y entrevista con los mismos, lectura de materiales referentes a la carrera como revistas, informes de investigaciones que den respuesta a problemas del territorio, materiales en soporte digital, etc. (Tiempo aproximado: 3 horas). Con estas actividades se procura que los estudiantes, mediante visitas a centros de trabajo, observación a profesionales en el ejercicio de la carrera, entrevistas con estos y lecturas de materiales referentes a la carrera, profundicen su conocimiento sobre la misma. La investi- 
gación se realizará en pequeños grupos, se elaborará un informe final y concluye con un taller vivencial, donde cada grupo comparte las experiencias resultantes de la investigación, con el grupo total de la clase.

3. Taller “¿Qué aspiro de mi vida profesional?”. Objetivo fundamental: esbozar aspiraciones profesionales para el futuro, a través de ejercicios. Se incluyen como actividades: exposiciones vivenciales de sus aspiraciones futuras donde aborden lo que esperan de la vida en relación con la carrera. (Tiempo aproximado: 2 horas).

También en esta etapa de implementación de la estrategia, y en aras de fomentar en el estudiantado el amor por la carrera y la motivación por la profesión, se podrán realizar actividades que contribuyan a fortalecer los motivos profesionales: realización de matutinos especiales una vez al mes donde se tribute al logro de los objetivos propuestos en el presente trabajo; convocatoria a eventos en la carrera donde participen de conjunto estudiantes y profesores, tributando así a la investigación de problemas sociales; edición de plegables que constituyan materiales de apoyo a la implementación de la estrategia educativa; incremento continuado de la biblioteca virtual de la carrera así como a través de diferentes actividades metodológicas en la carrera; demostraciones de la utilidad que tendría el incentivo de los motivos de la profesión a partir de la presentación de problemas profesionales desde la clase.

$\mathrm{Al}$ margen de estas acciones, las autoras consideran que son disímiles las actividades que se pueden realizar y que tributan a los objetivos propuestos. La estrategia educativa que se propone puede contribuir a incentivar la creatividad de profesores y estudiantes.

Quinta etapa: Evaluación y perfeccionamiento de la estrategia. Esta etapa comprende la aplicación de las técnicas aplicadas en otro momento y que permitan establecer si se han logrado alcanzar niveles superiores de motivación por la profesión en los estudiantes con la aplicación de la estrategia. Sobre la base de la pertinencia de las acciones concebidas de acuerdo a lo que arroje su evaluación, se introducirán los cambios necesarios para mejorar la efectividad de la estrategia.
Se evaluó la factibilidad de implementación de la estrategia educativa, utilizándose el método de expertos, en virtud de lo cual se corroboraron sus amplias potencialidades para introducirse en la vida universitaria. De igual manera, se ha iniciado la aplicación de la estrategia en el Centro Universitario Municipal y las primeras experiencias reportadas evidencian su efectividad para favorecer el desarrollo de la motivación profesional de los sociólogos en formación.

\section{Conclusiones}

La orientación profesional constituye un proceso de especial importancia en las condiciones de universalización de la educación superior en Cuba, signada por una enseñanza de tipo semipresencial. Se ha podido constatar que no ha sido estructurada coherentemente en la carrera de sociología del Centro Universitario del municipio Vertientes, en la provincia Camagüey, a pesar de su trascendencia para una formación exitosa del profesional.

Del mismo modo, desde la perspectiva científica, esta problemática ha sido insuficientemente estudiada. La motivación por la profesión es una de las aristas más significativas del proceso de orientación profesional, profundamente analizado por estudiosos de la temática en la educación superior cubana, resultando acogidos en el presente estudio los criterios de Fernando González Rey, Viviana González Maura y Jorge Luis del Pino Calderón, en conceptos como interés e intención profesional.

Se diseñó una estrategia educativa para incidir en la motivación profesional de los estudiantes de la carrera de sociología. La mentoría se alza como un recurso innovador y eficaz con antecedentes en el escenario universitario de otros países y adecuado a las condiciones de universalización de la educación superior cubana. La estrategia educativa que se presenta fue conformada para tributar al logro de niveles superiores de motivación por la profesión en el sociólogo en formación del Centro Universitario Municipal.

Los resultados preliminares obtenidos de la valoración de la estrategia educativa muestran su potencial utilidad para incidir favorablemente en la motivación profesional de los estudiantes de la carrera de sociología en condiciones de una enseñanza universitaria semipresencial. 


\section{Referencias}

Colunga Santos, S. (2009a). Tutoría universitaria y orientación educativa. Texto de Conferencia Magistral. $X$ Conferencia Internacional de Ciencias de la Educación, Camagüey. En ISBN 978-959-16-1123-9.

Colunga Santos, S. (2009b). Tutoría universitaria y diagnóstico psicopedagógico. Intervención en la Mesa Redonda: La tutoría en el contexto de la Educación Superior. X Conferencia Internacional de Ciencias de la Educación, Camagüey. En ISBN 978-959-16-1123-9.

González Maura, V. (1989). Nivel de integración de la motivación profesional (Tesis presentada en opción al grado científico de Doctor en Ciencias Psicológicas), Universidad de La Habana, La Habana.

González Maura, V. (1997). Diagnóstico y orientación de la motivación profesional. Pedagogía '97. IPLAC. La Habana.

González Maura, V. (2002). Orientación educativa-vocacional. Una propuesta metodológica para la elección y desarrollo profesional responsable. Curso en Congreso Internacional Universidad 2002, La Habana.

González Maura, V. (2002). ¿Qué significa ser un profesional competente? Revista Cubana de Educación Superior, 1(22), 45-53.

González Maura, V. (s/f). La orientación profesional un enfoque personológico para su instrumentación en la escuela. (Artículo mimeografiado).

González Rey, F. (1982). Motivación profesional en adolescentes y jóvenes. La Habana: Ed. Pueblo y Educación.

González Rey, F. (1989). La personalidad su educación y desarrollo. La Habana: Ed. Pueblo y Educación.

González Rey, F. (1995). Comunicación, personalidad y desarrollo. La Habana: Ed. Pueblo y Educación.

González Serra, D., González Maura, V., Brito Fernández, H. (1990). Motivación y orientación profesional. Congreso Internacional Pedagogía'90. La Habana.

Horruitiner, P. (2006). La universidad cubana: el modelo de formación. La Habana: Ed. Félix Varela.

Llerena Companioni, O. (2009). Estrategias potenciadoras del desarrollo de la motivación hacia la profesión en la educación Superior. Revista Iberoamericana de Educación, 3(48), 5-10.
Pino del, J. L. (1998). La orientación profesional en los inicios de la formación superior pedagógica. Una propuesta desde el enfoque problematizador (Tesis de grado Doctor en Ciencias Pedagógicas). ispejv, La Habana.

Pino del, J. L. (2009). La orientación profesional: una perspectiva desde el enfoque problematizador. La Habana: Memorias del Congreso Internacional Pedagogía 2009. 